\title{
Percepçáo de Professores em Relação ao Processamento Sensorial de Estudantes com Transtorno do Espectro Autista ${ }^{1}$ Teachers' Perception in ReLation to the Sensory Processing of Students WITH AUTISM SPECTRUM DISORDER
}

\author{
Rubiana Cunha MONTEIRO² \\ Camila Boarini dos SANTOS ${ }^{3}$ \\ Rita de Cássia Tibério ARAÚJO ${ }^{4}$ \\ Danielle dos Santos Cutrim GARROS 5 \\ Aila Narene Dahwache Criado ROCHA ${ }^{6}$
}

\begin{abstract}
RESUMO: Este estudo objetivou identificar a percepção dos professores em relaçáo ao processamento sensorial de estudantes com Transtorno do Espectro Autista (TEA). Participaram da pesquisa 19 professores de Educaçâo Infantil e Ensino Fundamental I de escolas públicas de um município do interior do estado de São Paulo, bem como seus 62 estudantes. A coleta de dados foi realizada entre os meses de setembro de 2018 e maio de 2019. Foi utilizada a avaliaçáo escalar Perfil Sensorial 2 de Acompanhamento Escolar, que avalia crianças e adolescentes a partir da perspectiva dos professores. A análise dos resultados ocorreu mediante categorias do instrumento. Em todas as categorias, Quadrantes (Exploração, Esquiva, Sensibilidade, Observação), Seçốes Sensoriais e Comportamentais (Auditivo, Visual, Tato, Movimentos e Comportamental) e Fatores Escolares (Fator Escolar 1, Fator Escolar 2, Fator Escolar 3 e Fator Escolar 4), a classificação de "Mais e Muito Mais que a Maioria dos Outros (as)" expressou a maior porcentagem, totalizando, em todos os aspectos avaliados pelo instrumento, 62,9\%. O estudo identificou que estudantes com TEA que apresentam um perfil caraterístico de disfunçóes de Integração Sensorial sofrem impacto da condição de estrutura e função corporal nos processos de ensino e de aprendizagem e na participaçáo em atividades dentro da sala de aula. Os resultados apontam para a importância das açóes do terapeuta ocupacional no ambiente escolar por meio do trabalho colaborativo com o professor, visto que os resultados em relaçáo ao perfil sensorial interferem diretamente no desempenho dos estudantes com TEA perante as demandas das atividades no contexto escolar.
\end{abstract}

PALAVRAS-CHAVE: Educação Especial. Processamento Sensorial. Escola. Terapia Ocupacional. Transtorno do Espectro Autista.

ABSTRACT: This study aimed to identify the perception of teachers in relation to the sensory processing of students with Autism Spectrum Disorder (ASD). Nineteen teachers of Early Childhood Education and early grades of Elementary Education from public schools in a municipality in the state of São Paulo, Brazil, participated in the research, as well as their 62 students. Data collection was carried out between the months of September 2018 and May 2019. A scale assessment of the Sensory Profile 2 of School Monitoring was used, which evaluates children and adolescents from the perspective of the teachers. The analysis of the results occurred through the categories of the instrument. In all categories, Quadrants (Sensory seeking, Sensory Avoiding, Sensory Sensitivity And Low Registration), Sensory and Behavioral Sections (Auditory, Visual, Tactile, Vestibular and Behavioral) and School Factors (School Factor 1, School Factor 2, School Factor 3 and School Factor 4 ), the classification of "More and Much More than the Majority of the Others" expressed the highest percentage, totaling, in all aspects assessed by the instrument $62.9 \%$. The study identified that students with ASD, who have a characteristic profile of Sensory Integration dysfunctions are impacted

\footnotetext{
${ }^{1}$ https://doi.org/10.1590/1980-54702020v26e0195

${ }^{2}$ Terapeuta Ocupacional graduada pela Universidade Estadual Paulista “Júlio de Mesquita Filho” (UNESP). E-mail: rubianamonteiroto@gmail.com. ORCID: https://orcid.org/0000-0003-3384-5352

${ }^{3}$ Terapeuta Ocupacional. Mestra em Educação pelo Programa de Pós-Graduação em Educação UNESP. E-mail: camilaboarini@ hotmail.com. ORCID: https://orcid.org/0000-0001-5594-0305

${ }^{4}$ Terapeuta Ocupacional. Doutora em Educação pela UNESP. Docente do Departamento de Fisioterapia e Terapia Ocupacional da UNESP e do Programa de Pós-Graduaçáo em Educação UNESP. E-mail: rita.araujo@unesp.br. ORCID: https://orcid. org/0000-0002-1463-1175

${ }^{5}$ Terapeuta Ocupacional. Doutora em Ciências da Saúde pela Faculdade de Ciências Médicas da Santa Casa de São Paulo. Docente do Departamento de Fisioterapia e Terapia Ocupacional da UNESP. E-mail: danielle.garros@unesp.br. ORCID: https://orcid. org/0000-0003-1525-0056

${ }^{6}$ Terapeuta Ocupacional. Doutora em Educaçáo pela UNESP. Docente do Departamento de Fisioterapia e Terapia Ocupacional da UNESP e do Programa de Pós-Graduação em Educação UNESP. E-mail: aila.rocha@unesp.br. ORCID: https://orcid. org/0000-0001-6186-875X
} 
by the condition of body structure and function in the teaching and learning processes and the participation in activities within the classroom. The results point to the importance of the occupational therapist's actions in the school environment through collaborative work with the teacher, since the results in relation to the sensory profile directly interfere with the performance of students with ASD in view of the demands of activities in the school context.

KEYWORDS: Special Education. Sensory Processing. School. Occupational Therapy. Autism Spectrum Disorder.

\section{INTRODUÇÃo}

A Integração Sensorial (IS) foi um termo utilizado pela primeira vez em 1963 por Anna Jean Ayres, terapeuta ocupacional. Ayres (1972) definiu IS como sendo um processo neurológico de organização das sensações provindas do corpo (tato, olfato, audição, visão, vestibular e proprioceptiva) e do ambiente, tornando possível a utilizaçáo correta do corpo no ambiente. Dessa forma, a IS caracteriza-se como um processo de input sensorial, seguido pelo processamento da informação no Sistema Nervoso Central (SNC) e output motor (resposta adaptativa) (Serrano, 2016).

A terapeuta ocupacional Winnie Dunn, 1997, propôs a definição de processamento sensorial, como uma forma de organizaçáo da IS, que busca a interaçáo entre o limiar neurológico e a autorregulaçâo da conduta do indivíduo. Dunn (1997) indica quatro modelos de quadrantes para o processamento sensorial, sendo eles: Exploração, Esquiva, Sensibilidade e Observação (Dunn, 1997, 2017; Metz et al., 2019).

Os quadrantes propostos por Dunn (1997) estáo relacionados à quantidade de estímulos sensoriais necessários para uma resposta neuronal (limiar neurológico) e a forma como os indivíduos se comportam para controlar suas necessidades (autorregulaçáo). No entanto, em cada quadrante apresentam-se características diferentes de comportamento (Dunn, 1997, 2017).

A criança ou adolescente que se encontra no quadrante de Exploração apresenta limiar neurológico elevado, mas possui estratégias de autorregulação ativa, representando o grau que uma criança obtém estímulo sensorial gerando novas ideias. Contrapondo-se a esse fato, o indivíduo que se enquadra em Observação apresenta um limiar neurológico elevado com autorregulação passiva, grau em que uma criança deixa de receber um estímulo sensorial.

Para o quadrante Sensibilidade, enquadram-se indivíduos que apresentam limiares neurológicos baixos e uma estratégia de autorregulação passiva, sendo o grau em que uma criança detecta um estímulo sensorial. No que se refere ao quadrante Esquiva, os indivíduos que nele se enquadram apresentam limiares neurológicos baixos e uma estratégia de autorregulaçấo ativa, significando o grau em que uma criança se incomoda com um estímulo sensorial (Dunn, 2017).

Quando o SNC não consegue ou tem dificuldades de processar as informações sensoriais do meio, emerge-se o que se chama Disfunções de IS. Essas disfunçóes são divididas em três categorias: Disfunçóes de Modulação Sensorial, Disfunçôes de Discriminação Sensorial e Disfunções Motoras de Base Sensorial (Serrano, 2016). 
A Disfunção de Modulação Sensorial caracteriza-se quando o indivíduo apresenta uma reatividade em excesso ou uma resposta insuficiente aos estímulos sensoriais, tendo dificuldade em responder apropriadamente à intensidade, natureza e grau do estímulo. Os indivíduos com Disfunção de Modulação Sensorial podem ser: 1) hiper-responsivos, quando exibem reaçôes exageradas aos estímulos; 2) hipo-responsivos, quando não respondem ou têm uma menor resposta aos estímulos; 3) Comportamentos de busca sensorial, quando necessitam de grande quantidade de informaçóes para ativar os sistemas sensoriais (Serrano, 2016).

Por um lado, os indivíduos que apresentam Disfunções de Discriminação Sensorial têm dificuldade em interpretar as informações de maneira correta, havendo, assim, uma falha na capacidade de dar significado às qualidades específicas dos estímulos. Por outro lado, os indivíduos que apresentam Disfunçôes Motoras de Base Sensorial podem ser subdivididos em indivíduos com Disfunção Motora, caracterizada pela dificuldade em estabilizar o corpo durante o movimento, e indivíduos com Dispraxia, caracterizados pela dificuldade em planejar, sequenciar e executar uma nova ação ou uma série de ações motoras (Serrano, 2016).

O Transtorno do Espectro Autista (TEA) é uma disfunção do neurodesenvolvimento caracterizada por alteraçóes comportamentais, de comunicação e de interação social. Estudos demonstram que cerca de $45 \%$ a $96 \%$ de indivíduos com TEA apresentam algum tipo de Disfunção de IS, esses indivíduos têm dificuldade para se adaptarem aos estímulos sensoriais que envolvem o ambiente, tendo tais alteraçóes impacto direto em sua participação social como, por exemplo, nas atividades escolares (Diretrizes de Atenção à Reabilitação da Pessoa com Transtornos do Espectro do Autismo, 2013; Howe \& Stagg, 2016; Metz et al., 2019).

A escola é caracterizada como um "ambiente natural”, que proporciona diversos estímulos, com diferentes frequências e graus de complexidade, sendo este um ambiente favorável para o desenvolvimento da criança. No período escolar, as crianças e os adolescentes com TEA, ou não, encontram na escola um ambiente propício para o input de informaçóes sensoriais, e é também nesse ambiente que as dificuldades no processamento sensorial são evidenciadas, o que pode gerar barreiras para a participação nas atividades que acontecem no contexto escolar (Piller, Fletcher, Pfeiffer, Dunlap, \& Pickens, 2017; Mills \& Chapparo, 2017). A Lei ${ }^{\circ}$ 12.764, de 27 de dezembro de 2012, institui a Política Nacional de Proteção dos direitos da pessoa com TEA e garante ao indivíduo o acesso à sala comum da rede regular de ensino e o direito, se houver necessidade, à acompanhante. Apesar da existência da lei, as especificidades do estudante com TEA, que diversas vezes são acentuadas pela Disfunção de IS, podem prover déficits de participação e o desempenho no contexto escolar, sendo necessárias algumas adaptaçóes no ambiente que atendam às demandas apresentadas pelo sujeito (Lei ${ }^{\circ}$ 12.764, 2012; Howe \& Stagg, 2016).

No ambiente escolar, o espaço físico e as pessoas envolvidas precisam ser preparados para receber um estudante de inclusão. Essa temática deve ser abordada considerando não apenas os estudantes e os professores, mas também os responsáveis pelas crianças, de modo a conscientizá-los do quanto o processo de inclusão é gratificante para a construção do conhecimento de seus filhos. Para o estudante com TEA, o ambiente escolar pode conter muitos estímulos, principalmente os visuais e auditivos, que podem ser perturbadores, tornando-se 
necessários cuidados em relação aos estímulos sensoriais presentes no contexto, a fim de que não prejudiquem a participação do estudante nas atividades (Brito \& Sales, 2014).

O terapeuta ocupacional, com um olhar biopsicosociocultural sobre o indivíduo, ao observar seus papéis ocupacionais, considera o contexto e o ambiente e intervém de forma a adequar a relação entre as demandas do ambiente e a capacidade funcional do indivíduo. No caso do estudante com TEA, são identificadas as barreiras que impedem a sua participação no ambiente escolar e a importância do terapeuta ocupacional se destaca, sobretudo na implementação de estratégias de IS que visam adequar o ambiente a fim de potencializar as habilidades desses indivíduos por meio de açóes realizadas juntamente ao professor (Mills \& Chapparo, 2017).

Após a busca de referenciais teóricos para esta pesquisa (Ashburner, Ziviani, \& Rodger, 2008; Kasari \& Smith, 2013; Weeks, Boshoff, \& Stewart, 2012), evidencia-se que a criança com TEA apresenta dificuldades no processamento sensorial; no entanto, há uma lacuna de estudos quanto ao conhecimento sobre a percepção de professores em relação ao perfil sensorial dos estudantes, bem como sobre as possíveis dificuldades que esses estudantes podem apresentar durante as atividades realizadas no contexto escolar. As evidências científicas estáo centradas em estudos de IS fora do contexto escolar, sendo ainda baixa a produçáo científica que evidencie o uso de intervençóes baseadas nas estratégias de IS no contexto escolar, principalmente com respeito a estudantes com TEA (Ashburner et al., 2008; Mills, Chapparo, \& Hinitt, 2016; Case-Smith, Weaver, \& Fristad, 2015). O cenário científico não é diferente no Brasil, pois não foi encontrado nenhum estudo sobre o assunto, o que confere destaque a esta proposta.

Dessa forma, a relevância deste estudo justifica-se pela necessidade de identificação da percepção dos professores em relação às habilidades das crianças, visando posteriormente a apresentação de propostas de intervenções baseadas no trabalho colaborativo entre os profissionais, terapeuta ocupacional e professor, no que concerne à IS no contexto escolar, direcionado à potencialização do acesso do estudante aos processos de ensino e de aprendizagem. Nessa perspectiva, o objetivo desta pesquisa foi, portanto, identificar a percepção dos professores em relação ao processamento sensorial dos estudantes com TEA.

\section{Método}

Este estudo foi submetido ao Comitê de Ética em Pesquisa da Faculdade de Filosofia e Ciências da Universidade Estadual Paulista "Júlio de Mesquita Filho" (UNESP) - Campus Marília, São Paulo, respeitando as prerrogativas da Resolução № 510, de 7 de abril de 2016, da Comissão Nacional de Ética em Pesquisa (CONEP), que versa sobre ética em pesquisa com seres humanos, tendo recebido parecer favorável, sob o Protocolo no 2.782.707 e CAAE:

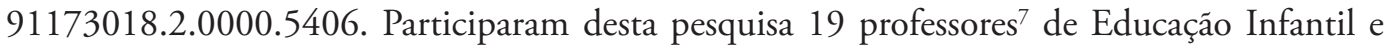
Ensino Fundamental I de escolas públicas de um município do interior do estado de São Paulo, bem como seus estudantes, sendo 62 no total.

\footnotetext{
${ }^{7}$ Alguns dos professores entrevistados trabalhavam no período da manhã e da tarde, sendo, dessa maneira, responsáveis por mais de um estudante.
} 
Os critérios de inclusão utilizados foram de que os estudantes participantes deveriam ter idade entre 3 anos e 0 meses a 14 anos e 11 meses, diagnóstico de TEA, sendo excluídos aqueles que apresentassem deficiências físicas, auditivas e visuais associadas ao TEA. A coleta de dados ocorreu entre os meses de setembro de 2018 e maio de 2019. Para a coleta, utilizou-se a avaliação escalar Perfil Sensorial 2 proposta por Winnie Dunn, que tem por objetivo comparar o desempenho da criança e/ou adolescente em relação a outros de sua mesma idade e identificar seu comportamento sensorial em diferentes contextos.

A avaliação utilizada é composta por cinco questionários (Perfil Sensorial 2 do Bebê, Perfil Sensorial 2 da Criança Pequena, Perfil Sensorial 2 da Criança, Perfil Sensorial 2 Abreviado, Perfil Sensorial 2 de Acompanhamento Escolar) e ofereceu um conjunto de ferramentas padronizadas para identificar os padróes de processamento sensorial da criança no contexto de vida diária. As informaçóes obtidas proporcionaram uma maneira de determinar como o processamento sensorial pode interferir em relação à participação da criança nos diferentes aspectos de sua vida social (Dunn, 2017).

Neste estudo, utilizou-se o questionário Perfil Sensorial 2 de Acompanhamento Escolar, que avalia crianças e adolescentes, a partir da perspectiva dos professores, composto por 44 itens sobre os estudantes e sua participação no contexto escolar. A primeira parte de cada formulário contém itens para descrever as respostas das crianças, termo utilizado pela avaliação, em relação às experiências sensoriais diárias no contexto escolar. As questões foram preenchidas pela pesquisadora, a partir das percepçóes dos professores que indicaram a frequência de respostas do estudante a diversas experiências sensoriais, usando uma escala de 5 pontos, variando o padrão de resposta da seguinte forma: Quase sempre equivale a 5 pontos; Frequentemente, a 4 pontos; Metade do tempo, a 3 pontos; Ocasionalmente, a 2 pontos; Quase nunca, a 1 ponto; e Não se aplica, a 0 ponto.

As pontuaçóes de corte da avaliação são baseadas nas médias e nos desvios padróes para cada pontuação resumida. Essas pontuaçóes proporcionam um sistema de classificação para categorizar a tendência de uma criança para comportamento específico. Esse sistema de classificação é composto por cinco categorias que refletem grupos específicos ao longo da curva em sino: Muito menos do que Outros(as); Menos do que Outros(as); Exatamente como a maioria dos(as) Outros(as); Mais do que Outros(as); e Muito mais do que Outros(as). Neste estudo, a análise dos dados ocorreu a partir do agrupamento de cinco categorias em três grupos: estudantes classificados como "Muito menos do que Outros(as) e Menos do que Outros(as)"; estudantes classificados como "Exatamente como a maioria dos(as) Outros(as)"; estudantes classificados como "Mais do que Outros(as) e Muito mais do que Outros (as)". Para a organização dos resultados, os dados foram tabelados por meio do Microsoft Office Excel. Para a análise dos resultados, foi utilizada a estatística descritiva, a fim de caracterizar o processamento sensorial por meio da avaliação de crianças com TEA.

\section{Resultados E DiscusSÃo}

Os resultados apresentados dizem respeito ao padrão do processamento sensorial de 62 estudantes. O Gráfico 1 e o Gráfico 2 caracterizam os estudantes deste estudo, contendo a idade e o gênero, respectivamente, dos participantes. 


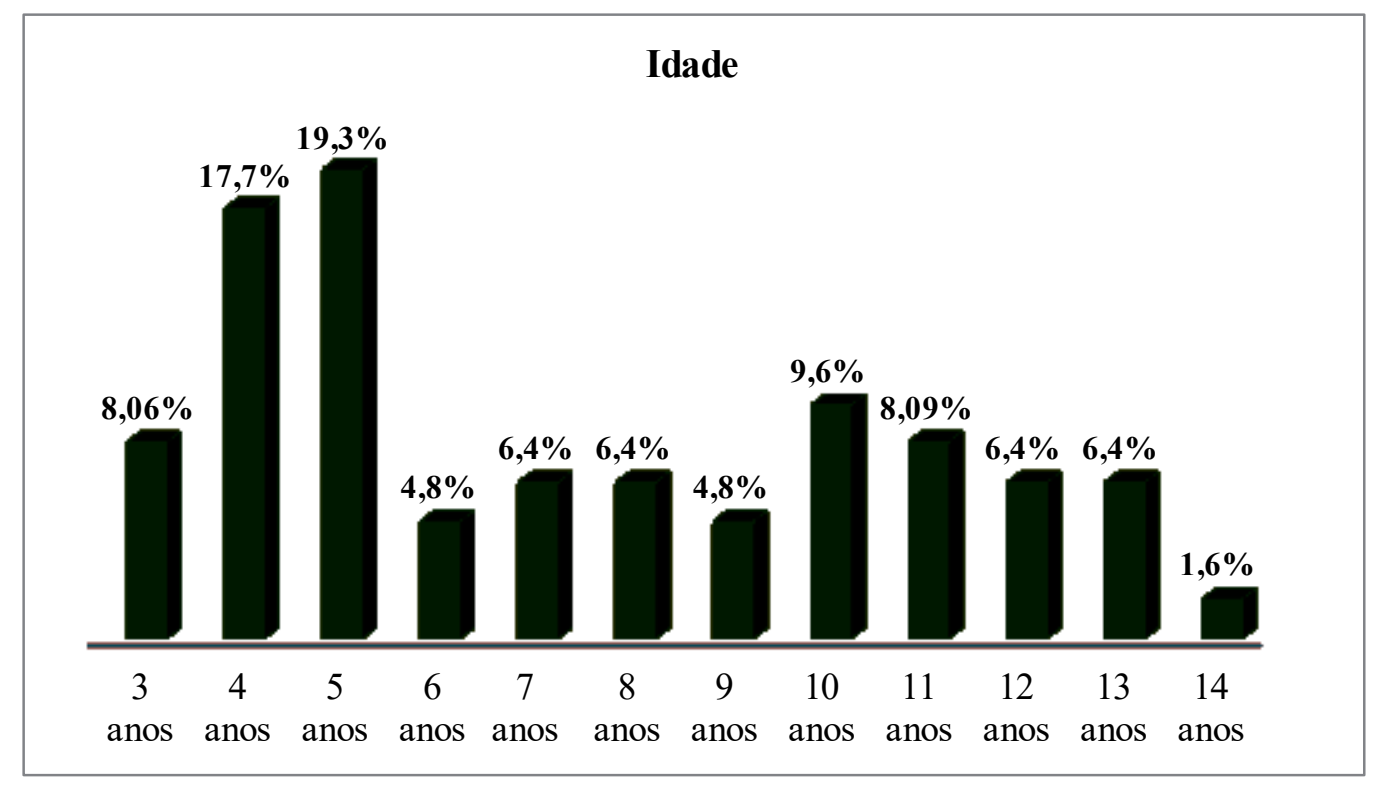

Gráfico 1. Idade dos participantes da pesquisa.

Fonte: Elaborado pelas autoras, 2019.

De acordo com o Manual de Orientação, desenvolvido pelo Departamento Científico de Pediatria do Desenvolvimento e Comportamento da Sociedade Brasileira de Pediatria ([SBP], 2019), o diagnóstico do TEA ocorre, em média, aos 4 e 5 anos de idade, dado significativo para o presente estudo, visto que a maioria dos estudantes estão enquadrados nessa faixa etária, representando, juntos, 37,09\% dos participantes (SBP, 2019).

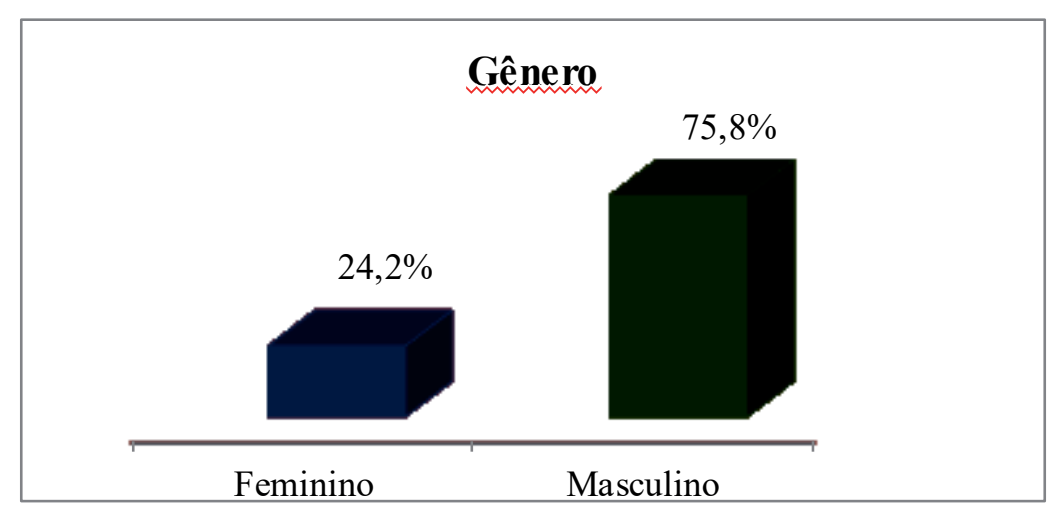

Gráfico 2. Gênero dos participantes da pesquisa.

Fonte: Elaborado pelas autoras, 2019.

Observa-se que, neste estudo, a maioria dos participantes é do gênero masculino, fator que converge com pesquisas publicadas relatando a prevalência do TEA por gênero, e, se- 
gundo a literatura, a prevalência do gênero masculino é quatro vezes maior do que a do gênero feminino (Christensen et al, 2016; Griesi-Oliveira \& Sertié, 2017).

Em relação aos padróes de processamento sensorial referente aos Quadrantes (Exploração, Esquiva, Sensibilidade, Observação), pode-se observar os resultados obtidos em relação à consideração da percepção dos professores na Figura 1.
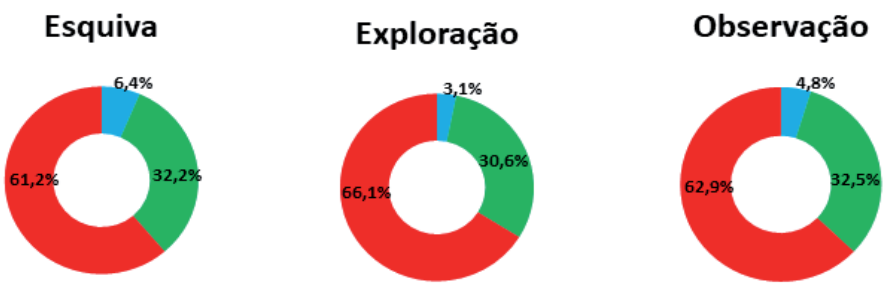

\section{Sensibilidade}

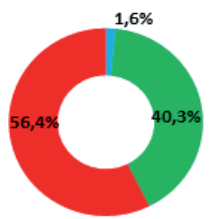

Legenda: Menos e Muito Menos do que a Maioria dos Outros(as)

Exatamente como a Maioria dos Outros(as)

Mais e Muito Mais do que Maioria dos Outros(as)

Figura 1. Quadrantes: Exploração, Esquiva, Sensibilidade e Observação.

Fonte: Elaborada pelas autoras, 2019.

Observa-se que, nesta pesquisa, o quadrante com maior número de crianças enquadradas em "Mais e Muito Mais do que a Maioria dos Outros(as)" é o de Exploração (Figura 1 ), representados com $66,1 \%$ dos estudantes. Dessa forma, os estudantes classificados em tal pontuação no quadrante Exploração têm interesse em explorar o ambiente, buscando oportunidades para aumentar a entrada de estímulos sensoriais em todas as atividades. É fundamental considerar que a busca constante por estímulos pode colocar a criança em risco, visto que, durante as brincadeiras no parquinho da escola, por exemplo, elas não podem buscar acentuadamente determinados estímulos sem se preocupar com a sua segurança (Dunn, 2017).

O estudo de Mills e Chapparo (2017) teve por objetivo captar as percepçóes dos professores em relação ao uso de um cronograma de atividades na sala de aula. Os pesquisadores entrevistaram 19 professores de estudantes com TEA e relatam que o comportamento explorador dos estudantes com TEA é agravado por inputs visuais e auditivos, fato que justifica a média de 67,1\% dos estudantes deste estudo estarem classificados em "Mais e Muito Mais do que a Maioria dos Outros(as)" tanto no quadrante de Exploração (Figura 1), quanto nas seçôes sensoriais Auditivo e Visual (ver Figura 3 mais adiante). 
Nos quadrantes de Esquiva e Observação, os resultados referentes aos estudantes que se classificam em "Exatamente como a Maioria dos Outros(as)" se igualam, representando 32,25\%. O comportamento de Esquiva está relacionado à participação dos estudantes nas atividades. O estudo de Piller e Pfeiffer (2016) teve como objetivo explorar a relação entre as características sensoriais do ambiente pré-escolar a partir da perspectiva de 13 profissionais entre professores e terapeutas ocupacionais que trabalhavam com crianças com TEA, por meio do "Questionário do ambiente sensorial da participação" (PSEQ-TV). Os resultados desse estudo identificaram que as crianças com um comportamento "mais ou muito mais do que a maioria" evitaram atividades que requeriam maior estimulação sensorial, como, por exemplo, as atividades que envolviam a entrada tátil, sendo a participação das crianças dentro dessas tarefas afetada pelo input sensorial. Tal dado corrobora o presente estudo, tendo em vista as alteraçóes sensoriais tanto no comportamento de Esquiva (Figura 1), quanto no Tato (ver Figura 2 mais adiante).

Outro estudo que identificou as dificuldades de crianças com TEA que apresentam o comportamento de esquiva mais evidente do que seus pares foi realizado por Ashburner et al. (2008), tendo como objetivo explorar as associaçóes entre processamento sensorial, emocional, comportamental e os resultados educacionais de crianças com TEA. Nesse estudo, 28 crianças com TEA foram comparadas a 51 crianças com desenvolvimento do processamento sensorial sem alteraçóes. Os resultados que se relacionaram ao contexto escolar indicaram que crianças com comportamentos de esquiva preferem ambientes sensoriais com poucos estímulos, podendo apresentar dificuldades em prestar atenção em instruçóes verbais quando se tem a presença de outros estímulos auditivos, o que interfere no desempenho acadêmico do estudante, fazendo com que ele seja insuficiente (Ashburner et al., 2008; Dunn, 2017).

Os resultados do estudo de Ashburner et al. (2008) corroboram com os resultados identificados nesta presente pesquisa, visto que, no estudo dos autores, foi identificado que $72 \%$ das crianças com TEA têm o padrão sensorial de Observação e, neste estudo, a porcentagem foi de $62,9 \%$. Tal padrão pode pressupor um pior desempenho acadêmico, tendo em vista que estudantes observadores podem demorar a detectar um estímulo, realizando uma busca constante para aumentar seus limites de inputs sensoriais. Nesse caso, Ashburner et al. (2008) e Dunn (2017) indicam a utilização de pistas como estratégia a fim de manter o estudante envolvido na atividade proposta pelo professor.

No quadrante Sensibilidade, a porcentagem de estudantes que apresentaram disfunção no processamento sensorial, classificadas em "Mais e Muito Mais do que a Maioria dos Outros(as)" representam 56,4\%, dado que corrobora a pesquisa realizada por Simpson, Adams, Alston-Knox, Heussler, \& Keen (2019), a qual identificou subtipos sensoriais em crianças com TEA usando o Perfil Sensorial 2, sendo este respondido pelos cuidadores das crianças. O estudo citado (Simpson et al., 2019) obteve resultado semelhante ao deste estudo, tendo em vista que, na perspectiva dos pais, as crianças com TEA apresentaram um comportamento "Mais e Muito Mais do que a Maioria dos outros(as)" em 65,7\% dos casos, corroborando a percepção dos professores analisada na presente pesquisa.

Os resultados obtidos nesta pesquisa têm implicações no ambiente escolar, que deve estar adequadamente organizado para favorecer o desempenho da criança, visto que os estímu- 
los do ambiente podem causar uma auto desregulação no estudante com TEA, comprometendo o ensino e a aprendizagem (Dunn, 2017).

A Figura 2 apresenta os resultados a respeito das Seçóes Sensoriais e Comportamentais (Auditivo, Visual, Tato, Movimentos e Comportamental) da percepção dos professores em relação a seus estudantes.
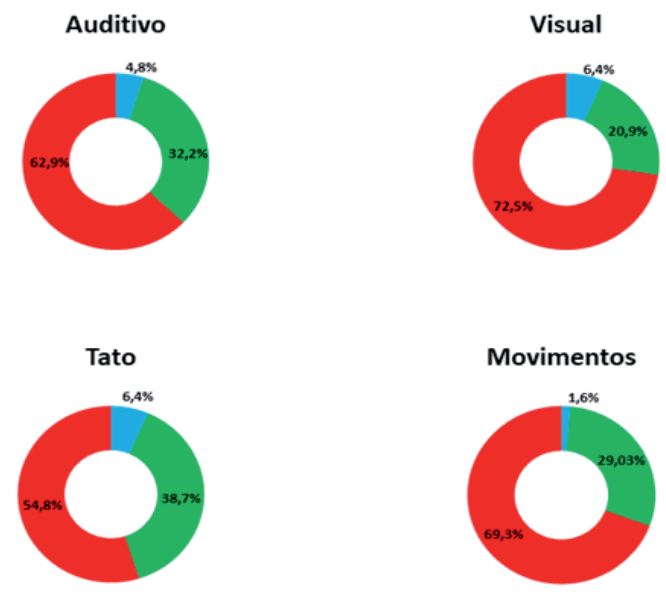

Comportamento

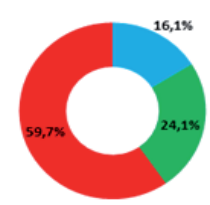

Legenda: Menos e Muito Menos do que a Maioria dos Outros(as)

- Exatamente como a Maioria dos Outros(as)

- Mais e Muito Mais do que a Maioria dos Outros(as)

Figura 2. Seções sensoriais e comportamentais: auditivo, visual, tato, movimentos e comportamental.

Fonte: Elaborada pelas autoras, 2019.

Em relação à seção sensorial Auditivo (Figura 2), estudantes que se enquadram nas categorias "Mais e Muito Mais do que os Outros(as)" podem ser incomodados por estímulos auditivos comumente presentes no ambiente escolar, tendo como consequência a ação de evitar as tarefas exigidas, estando, neste estudo, 62,9\% dos estudantes classificados nessa categoria.

Os estudantes enquadrados em "Mais e Muito Mais do que a Maioria dos Outros(as)", na seção sensorial Auditivo (Figura 2) e nos quadrantes apresentados na Figura 2 de Comportamento $(59,67 \%)$ e Movimentos (69,35\%) se aproximam, fator que corrobora o estudo de Piller e Pfeiffer (2016), em que os professores relataram que estudantes com sensibilidade auditiva demonstravam uma aversão tão forte perante o eco em sala de aula que o comportamento no ambiente se modificava, tendo como consequência uma movimentação di- 
ferenciada, podendo, em alguns casos, ficarem em posição fetal no chão sempre que o professor começava a falar (Piller \& Pfeiffer, 2016).

Howe e Stagg (2016) investigaram as experiências de adolescentes com TEA enquanto estavam em uma sala de aula, utilizando uma técnica qualitativa para aceder às experiências subjetivas dos participantes em questóes sensoriais dentro desse ambiente. Para a coleta de dados, os autores utilizaram um questionário estruturado que exigia respostas escritas. Os resultados do estudo citado demonstrou que todos os participantes apresentaram limiares altos em relação ao padrão auditivo, o que afeta a aprendizagem do estudante com TEA no contexto escolar, sendo este o único aspecto considerado por todos os estudantes como ruim em seu envolvimento escolar (Howe \& Stagg, 2016).

O estudo de Giacardy et al. (2018) examinou a relação entre sintomas de modulação sensorial e comportamentos mal adaptativos em um grupo de crianças com TEA. Para a coleta de dados, os autores utilizaram como proposta metodológica a observação transversal, em que as características de modulação sensorial foram avaliadas usando o Perfil Sensorial Abreviado de Dunn (2017), e os comportamentos adaptativos e o quociente social foram avaliados pela Escala de Comportamento Adaptativo proposta por Vineland. Foi identificado que as crianças que apresentaram disfunçóes sensoriais tiveram interferência em seus comportamentos adaptativos nas atividades de vida diária como, por exemplo, dificuldades para vestir-se e ir ao banheiro, tarefas que são realizadas pelo estudante no contexto escolar e que provavelmente necessitaram de planejamento prévio e maior apoio externo para serem efetivadas (Giacardy et al., 2018).

Identificou-se, neste estudo, que, na seção sensorial Visual, ocorreu a maior prevalência de estudantes classificados como "Mais e Muito Mais do que a Maioria dos Outros(as)", com 72,5\%, como pode ser observado na Figura 3. Tanto no estudo de Piller e Pfeiffer (2016) quanto no de Ashburner et al. (2008) foi observado que 43\% das crianças apresentavam disfunçóes no processamento visual. Os professores entrevistados nos dois estudos identificaram algumas estratégias para aumentar a participação em sala de aula de seus estudantes, sendo uma das estratégias descritas por esses professores a mudança na iluminaçáo da sala, escurecendo ou apagando as luzes.

Observa-se que os resultados obtidos pelos estudantes no quadrante Observação (Figura 1) e na seção sensorial Auditiva (Figura 2) se igualam no que diz respeito aos classificados em "Mais e Muito Mais do que a Maioria dos Outros(as)", representando 62,9\% dos participantes. Tal dado corrobora o estudo realizado por Ashburner et al. (2008) em que os professores identificaram que estudantes com padrão sensorial de observação têm um pior desempenho acadêmico, tendo em vista a possível dificuldade de processar informaçóes que chegam por meio de estímulos auditivos. Esses estudantes podem apresentar como comportamento a busca acentuada por estímulos auditivos que apresentam repetiçóes previsíveis para que, dessa forma, consigam interpretá-las (Ashburner et al., 2008).

O estudo de Mills e Chapparo (2017) reconheceu que a utilização da intervenção por meio da IS, implementadas por terapeutas ocupacionais, foi eficiente para a diminuição dos comportamentos desafiadores apresentados por estudantes com TEA durante as atividades realizadas no contexto escolar (Mills \& Chapparo, 2017). 
Os Fatores Escolares permitem o cruzamento de informaçôes dos padróes de processamento sensorial do estudante com as características de aprendizagem eficientes para serem utilizadas no contexto escolar, sendo estes respondidos segundo a perspectiva do professor (Dunn, 2017). A Figura 3 representa as respostas relacionadas aos Fatores Escolares (Fator Escolar 1, Fator Escolar 2, Fator Escolar 3 e Fator Escolar 4). Cada resultado obtido leva em consideração a percepção dos professores sobre seus estudantes, independentemente do gênero da criança.

\section{Fator Escolar 1}

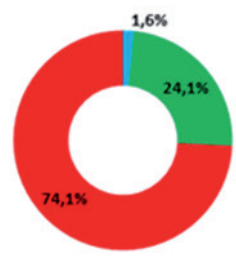

Fator Escolar 3

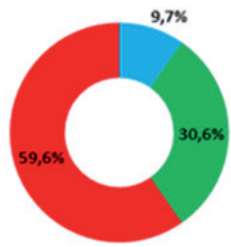

Fator Escolar 2

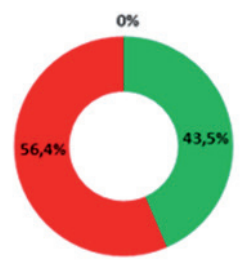

Fator Escolar 4

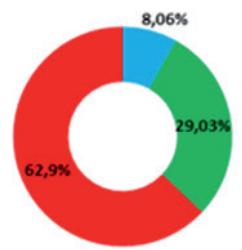

Legenda: Menos e Muito Menos do que a Maioria dos Outros(as)

- Exatamente como a Maioria dos Outros(as)

- Mais e Muito Mais do que Maioria dos Outros(as)

Figura 3. Fatores Escolares (Fator Escolar 1, Fator Escolar 2, Fator Escolar 3 e Fator Escolar 4) Fonte: Elaborada pelas autoras, 2019.

$\mathrm{Na}$ categoria Fator Escolar 1, que representa a necessidade de o estudante receber apoio do professor e/ou mediador escolar para participar das atividades em sala de aula, a classificação "Mais e Muito Mais do que a Maioria dos Outros(as)" prevalece com 79,1\% dos participantes. Estudantes enquadrados nessa pontuaçáo necessitam de planejamento e de adaptação dos estímulos sensoriais para favorecer a aprendizagem. De acordo com Dunn (2017), os professores caracterizam as crianças com essas atitudes como aquelas que necessitam de mais atenção para realizar as tarefas propostas em sala de aula de maneira efetiva, necessitando ora de redirecionamento, ora de orientaçôes para a realização das atividades propostas.

Mills e Chapparo (2017) descrevem que, apesar de os professores identificarem as dificuldades em relação ao processamento sensorial de estudantes com TEA, os professores relataram que não possuem conhecimentos necessários para selecionar recursos que promovam a participação do estudante de forma eficaz. Os mesmos autores destacaram ser importante 
o estabelecimento de parcerias entre terapeutas ocupacionais e professores para a avaliação e intervenção de estudantes com disfunção de processamento sensorial, uma vez que essa condição tem impacto nas atividades de aprendizagem específicas no contexto escolar (Mills \& Chapparo, 2017).

O Fator Escolar 2 reflete a consciência e a atenção do estudante dentro da sala de aula. Nesse fator, nenhum estudante foi classificado na categoria "Menos e Muito Menos do que a Maioria dos Outros(as)". Ainda em relação ao Fator escolar 2, a classificação "Mais e Muito Mais do que a Maioria dos Outros(as)" representa 56,4\% dos estudantes. No ponto de vista dos professores, tais estudantes são bastante atentos dentro da sala de aula, apesar de, às vezes, seus níveis de atenção interferirem em sua capacidade de aderir às atividades de aprendizagem propostas (Dunn, 2017).

Segundo Grandin (2014), crianças com TEA apresentam dificuldades nos mecanismos neurológicos que controlam a capacidade de dividir a atenção entre diferentes estímulos. Os estímulos sensoriais desconfortáveis podem direcionar a concentração para fora dos principais elementos do ambiente, como, por exemplo, desviando a atenção durante a apresentação da aula. Os professores que participaram da pesquisa de Mills e Chapparo (2017) relataram acreditar que a utilização de intervençóes de IS tiveram um impacto positivo sobre a capacidade de atenção e concentração do estudante durante as atividades do contexto escolar. No estudo citado, as atividades realizadas com IS pelos professores ocorreram no ambiente escolar e foram adequadas ao cronograma de atividades do professor (Grandin, 2014; Howe \& Stagg, 2016; Mills \& Chapparo, 2017).

O Fator Escolar 3 diz respeito à tolerância do estudante dentro do ambiente de aprendizagem. Dos estudantes que participaram da pesquisa, 59,67\% deles foram enquadrados em "Mais e Muito Mais do que a Maioria dos Outros(as)". Por ser um fator em que se espera limiares baixos, os estudantes com essa pontuação podem ficar sobrecarregados rapidamente em ambientes de aprendizagem, apresentando comportamento que interfere em sua capacidade de compreender instruçóes, realizar trabalhos independentemente ou cooperar com outros estudantes da sala (Dunn, 2017).

Mills e Chapparo (2017) relatam que mudanças no contexto e no ambiente do estudante com TEA interferem na execução das atividades propostas em sala de aula. Os professores do estudo citado observaram que há um desafio para utilizar estratégias que facilitem as habilidades dos estudantes, a fim de que concluam as atividades corretamente e de forma consistente. Piller e Pfeiffer (2016) corroboram os achados de Mills e Chapparo (2017), na medida em que os participantes de seu estudo descreveram que mudanças na rotina de sala de aula muitas vezes fazem com que o estudante busque por mais estímulos sensoriais, o que acontece com crianças classificadas em "Mais e Muito Mais do que a Maioria dos Outros(as)”, caracterizando a maior prevalência de classificação de estudantes com TEA deste estudo.

Estudantes com alteraçóes no Fator Escolar 3 necessitam de um ambiente de aprendizagem controlado, visto sua reatividade e exigência extrema. Os professores participantes do estudo de Piller e Pfeiffer (2016) relataram que a adesão às rotinas estruturadas são componentes essenciais para apoiar a participação das crianças, indicando diferentes estratégias, como lembretes visuais e verbais da mudança na rotina. Uma sala de aula estruturada em torno 
das necessidades sensoriais dos estudantes é um elemento importante para uma participação bem sucedida dos estudantes com TEA no ambiente de aprendizagem (Dunn, 2017; Piller \& Pfeiffer, 2016).

Já o Fator Escolar 4 representa a disponibilidade do estudante para a aprendizagem, e, nesse fator, os estudantes que se enquadram em "Exatamente como a Maioria dos Outros(as)" representam 29,03\% dos participantes, e os estudantes classificados em "Mais e Muito Mais do que a Maioria dos Outros(as)" representam 62,9\% dos participantes nessa classificação. Estudantes com padróes inesperados nesse fator perdem oportunidades de participar e aparentam desinteresse durante as tarefas de aprendizagem, necessitando de uma retomada das instruçóes e do conteúdo da atividade proposta. É fundamental que os profissionais que atuam com esse estudante identifiquem a quantidade ideal de estimulação sensorial para permitir a participação efetiva do estudante em contexto escolar (Dunn, 2017).

Neste estudo, observa-se que, em todas as categorias do instrumento - Quadrantes (Figura 1), Seçóes Sensoriais e Comportamentais (Figura 2) e Fatores Escolares (Figura 3) -, a classificação de "Mais e Muito Mais do que a Maioria dos Outros(as)" expressa a maior prevalência de classificação, totalizando, em todos os aspectos avaliados pelo instrumento, 62,9\%. Esse resultado corrobora o estudo de Simpson et al. (2019), no qual se identificou que os participantes apresentaram uma classificação de "Mais e Muito Mais do que a Maioria dos Outros(as)" em todas as categorias do instrumento, sendo em ambos os estudos a maior pontuação obtida.

Os resultados deste estudo demonstram evidência quanto à probabilidade de grande parte dos estudantes com TEA apresentarem disfunçóes de IS e, consequentemente, a necessidade do encaminhamento desses estudantes para avaliação e possível intervenção baseada na Teoria de IS proposta por Ayres (1972). Atualmente, no Brasil, a formação de profissionais para intervençóes que utilizam a teoria de IS proposta por Ayres é oferecida para profissionais da área de terapia ocupacional, assim como propóe a Associação Brasileira de Integração Sensorial ([ABIS], 2013).

Há, no entanto, a necessidade de um trabalho direcionado ao uso de intervenções baseadas em estratégias de IS no contexto escolar, a fim de ajudar estudantes com TEA a ter acesso aos processos de ensino e de aprendizagem, considerando que as condiçóes ambientais afetam diretamente o desempenho ocupacional desse estudante. A Resolução No 500, de 26 de dezembro de 2018, do Conselho Federal de Fisioterapia e Terapia Ocupacional (COFFITO), reconhece a especialidade de Terapia Ocupacional no Contexto Escolar, sendo o trabalho colaborativo entre terapeutas ocupacionais e professores significativo, visto que, por meio dele, se estabelece uma relação benéfica, a fim de buscar estratégias para a redução dos comportamentos indesejáveis.

Os resultados deste estudo bem como as demais evidências da literatura (Ashburner et al., 2008; Dunn, 2017; Mills \& Chapparo, 2017; Piller \& Pfeiffer, 2016) permitem identificar três domínios que justificam e direcionam a proposta de trabalho colaborativo entre terapeutas ocupacionais e profissionais da educação envolvendo intervenções de IS no contexto escolar: 
1. A compreensão, a análise e a intervenção no ambiente físico: é fundamental identificar as informaçóes sensoriais que envolvem o ambiente escolar e propor modificaçóes ambientais adequadas às habilidades do estudante com TEA. Como exemplo, é possível citar o cuidado em relação ao nível de ruídos, à iluminação do ambiente, às texturas que envolvem a atividade, ao cheiro do ambiente, ao excesso de estímulos visuais, dentre outras características sensoriais que podem estar presentes.

2. A compreensão, a análise e a intervenção no ambiente social: envolve a atenção aos relacionamentos e as expectativas do estudante com TEA em relação às diferentes pessoas presentes no contexto escolar. Para isso, há necessidade de: a) identificar as pessoas que são referências para o estudante; b) capacitar os profissionais para reconhecer as habilidades de estudantes com TEA em diferentes atividades e a relação dessas habilidades com a IS (atividades em sala, no parque, na quadra, no recreio e em outros ambientes escolares; c) trabalhar as interaçóes entre o estudante e seus pares, considerando as expectativas para a faixa etária, os papéis e a rotina e também levando em conta as possíveis interferências no desempenho do estudante com TEA relacionadas ao processamento sensorial da criança.

3. A implementação de recursos e estratégias: cabe ao terapeuta ocupacional realizar a análise das atividades presentes no contexto escolar e, posteriormente, junto ao professor planejar e implementar recursos e estratégias baseadas na teoria de IS que podem ampliar o desempenho do estudante. Um exemplo desse domínio seria o uso de fones de ouvido para a diminuição de ruídos que envolvem o ambiente, a implementação de atividades específicas que visam aumentar ou diminuir o nível de alerta do estudante, o uso de recursos visuais específicos durante as atividades, a necessidade do uso da Comunicação Suplementar e Alternativa, entre outras propostas que atendam às demandas individuais de cada estudante.

\section{Conclusāo}

Este estudo teve por objetivo identificar a percepção dos professores em relação ao processamento sensorial dos estudantes com TEA. Para isso, utilizou-se como instrumento de pesquisa o Perfil Sensorial 2 de Acompanhamento Escolar, que avalia crianças e adolescentes a partir da perspectiva dos professores. Os resultados deste estudo demonstraram que, na classificação dos estudantes com TEA e o perfil característico para Disfunções de IS, prevaleceu a indicação de hiper-responsivas. Esse resultado sinaliza a importância de cuidados quanto à adequação do ambiente para a realização de atividades, mediante a implementação de intervençóes baseadas em estratégias de IS para os estudantes, a fim de melhorar a participação e o desempenho desses alunos nas atividades escolares.

Sugere-se, portanto, que haja o trabalho colaborativo entre o terapeuta ocupacional e os profissionais da educação para realizar as modificaçóes ambientais e de rotina relacionadas ao processamento sensorial, buscando apoiar a autorregulação e a criação de oportunidades para facilitar a participação do estudante com TEA no contexto escolar. Além disso, evidencia-se a necessidade de políticas públicas que garantam atuação do terapeuta ocupacional no contexto escolar, garantindo, assim, não apenas intervençóes com enfoque clínico, que já ocorrem no 
ambiente externo, mas também o trabalho colaborativo entre o terapeuta ocupacional e os profissionais da educação. Sugere-se em estudos futuros a implementação e a análise de intervençóes por meio do trabalho colaborativo no contexto escolar, baseadas em estratégias de IS que considerem os domínios identificados neste estudo.

]

\section{REFERENCIAS}

Ashburner, J., Ziviani, J., \& Rodger, S. (2008). Sensory processing and classroom emotional, behavioral, and educational outcomes in children with autism spectrum disorder. American Journal of Occupational Therapy, 62(5), 564-573. https://doi.org/10.5014/ajot.62.5.564

Associação Brasileira de Integração Sensorial. (2013). Estatuto Social da Associação Brasileira de Integração Sensorial. Recuperado em 31 de março de 2020 de https:/www.integracaosensorialbrasil.com.br/ estatuto-texto

Ayres, A. J. (1972). Sensory integration and learning disorders. Los Angeles, CA: Western Psychological Services.

Brito, A., Sales, N. B. (2014). TEA e inclusão escolar: um sonho mais que possível. São Paulo: Edição do Autor.

Case-Smith, J., Weaver, L. L., \& Fristad, M. A. (2015). A systematic review of sensory processing interventions for children with autism spectrum disorders. Autism 19(2), 133-148.

Christensen, D. L., Braun, K. V. N., Baio, J., Bilder, D., Charles J., Constantino, J. N. ... \& YearginAllsopp, M. (2018). Prevalence and characteristics of Autism Spectrum Disorder among children aged 8 years - autism and developmental disabilities monitoring network, 11 Sites, United States, 2012. MMWR Surveill Summ, 65(13), 1-23.

Diretrizes de Atenção à Reabilitação da Pessoa com Transtornos do Espectro do Autismo. (2013). Brasília: Ministério da Saúde, Secretaria de Atenção à Saúde.

Dunn, W. (1997). The impact of sensory processing abilities on the daily lives of young children and their families: A conceptual model. Infants Young Child, 9(4), 23-35.

Dunn, W. (2017). Perfil Sensorial 2: abordagem baseada em pontos fortes para avaliação e planejamento. São Paulo: Pearson Clinical.

Giacardy, P., Viellard, M., Chatel, C., Jourdan, E., Avenel, E., Elissalde, S. ... \& Poinso, F. (2018). Troubles de la modulation sensorielle et difficulte's adaptatives dans les troubles du spectre de l'autisme. Elsevier Masson SAS, 315-321.

Grandin, T. (2014). Eletronic publishing at IIDC: An inside view of autism. Indiana Resource Center for Autism. Recuperado em 11 de outubro de 2019 de http://www.iidc.indiana.edu/index. php?pageId $=595$.

Howe, F. E. J., \& Stagg, S. D. (2016). How sensory experiences affect adolescents with an autistic spectrum condition within the classroom. J. Autism Dev Disord, 20, 1656-1668.

Kasari, C., \& Smith, T. (2013). Interventions in schools for children with autism spectrum disorder: Methods and recommendations. Autism: The International Journal of Research and Practice, 254-267.

Lei No 12.764, de 27 de dezembro de 2012. Institui a Política Nacional de Proteção dos Direitos da Pessoa com Transtorno do Espectro Autista; e altera o $₫ 3^{\circ}$ do art. 98 da Lei $n^{\circ} 8.112$, de 11 de 
dezembro de 1990. Recuperado de http://www.planalto.gov.br/ccivil_03/_Ato2011-2014/2012/ Lei/L12764.htm

Metz, A. E., Bolling, D., DeVore, A., Holladay, H., Liao, J. F., \& Vlutch, K. V. (2019). Dunn’s model of sensory processing: an investigation of the axes of the four-quadrant model in healthy adults. Brain Sciences, 9(35), 1-15.

Mills, C., \& Chapparo, C. (2017). Listening to teachers: Views on delivery of a classroom based sensory intervention for students with autismo. Australian Occupational Therapy Journal, 31, 15-24.

Mills, C., Chapparo, C., \& Hinitt, J. (2016). The impact of an in-class sensory activity schedule on task performance of children with autism and intellectual disability: A pilot study. British Journal of Occupational Therapy, 79, 530-539.

Griesi-Oliveira, K., Sertié, A. L. (2017). Transtornos do espectro autista: um guia atualizado para aconselhamento genético. J. Einstein, 15(2), 233-238.

Piller, A., \& Pfeiffer, B. (2016). The sensory environment and participation of preschool children with Autism Spectrum Disorder. OTJR: Occupation, Participation and Health, 36(3), 103-111.

Piller, A., Fletcher, T., Pfeiffer, B., Dunlap, K., \& Pickens, N. (2017). Reliability of the participation and sensory environment questionnaire: teacher version. J Autism Dev Disord, 47, 3541-3549.

Resolução no 500, de 26 de dezembro de 2018. Reconhece e disciplina a especialidade de Terapia Ocupacional no Contexto Escolar, define as áreas de atuação e as competências do terapeuta ocupacional especialista em Contexto Escolar e dá outras providências. Recuperado de http:// abmes.org.br/arquivos/legislacoes/Resolucao-COFFITO-500-2018-12-26.pdf

Serrano, P. (2016). A Integração Sensorial: no desenvolvimento e aprendizagem da criança. Lisboa: Papa Letras.

Simpson, K., Adams, D., Alston-Knox, C., Heussler, H. S., \& Keen, D. (2019). Exploring the sensory profiles of children on the autism spectrum using the Short Sensory Profile-2 (SSP-2). Journal of Autism and Developmental Disorders, 49, 2069-2079.

Sociedade Brasileira de Pediatria (2019). Transtorno do Espectro do Autismo. Brasil: SBP.

Weeks, S., Boshoff, K., \& Stewart, H. (2012). Systematic review of the effectiveness of the Wilbarger protocol with children. Pediatric Health, Medicine and Therapeutics, 3, 79-89.

Recebido em: 16/12/2019

Reformulado em: 01/05/2020

Aprovado em: 06/07/2020 